\title{
Excitation of plasmon and guided-mode resonances in thin film silicon solar cells
}

\author{
F.-J. Haug, K. Söderström, A. Naqavi, C. Battaglia and C. Ballif \\ Ecole Polytechnique Fédérale de Lausanne (EPFL), Institute of Microengineering (IMT), \\ Photovoltaics and Thin Film Electronics Laboratory, \\ Rue A.-L. Breguet 2, CH-2000 Neuchâtel, Switzerland
}

\begin{abstract}
Thin film silicon solar cells are an attractive option for the production of sustainable energy but their low response at long wavelengths requires additional measures for absorption enhancement. The most successful concepts are based on light scattering interface textures whose understanding is greatly facilitated by considering a superposition of periodic textures that diffract the light into oblique angles, ideally beyond the critical angle of total internal reflection. Because the thickness of the active layers is on the same scale as the wavelength, interference of diffracted waves gives rise to resonance phenomena. We discuss the absorption enhancement in terms of a perturbation approach using the modal structure of a corresponding device with flat interfaces.
\end{abstract}

\section{INTRODUCTION}

Against the background of global warming and dwindling of traditional energy resources, the access to clean and sustainable energy has become a major issue for humanity. Thin film silicon solar cells can make a vital contribution because they rely on abundant materials and their manufacturing technology is well established. Owing to relatively poor charge transport, the first report on amorphous silicon solar cells by Carlson et.al noted already that the devices must be kept thin, ideally thinner than the absorption length for light with energies just above the optical band gap [1]; therefore, most modern device architectures are based on a concept of Deckman et al. where light scattering at textured interfaces enhances the effective light path within the absorber film [2]. When light is scattered beyond the critical angle of total internal reflection, it propagates back and forth within this layer. If the light does not get scattered out of the device, it will eventually be absorbed. Based on weak absorption, ideal Lambertian scattering and a statistically uniform distribution of energy among all available radiation modes inside and outside of a slab of material, Yablonovitch et al. proposed that the absorption enhancement cannot exceed an upper limit of $4 n^{2}$, where $n$ is the refractive index of the absorber layer [3]. Sheng et al. noted that it should be possible to redistribute the density of modes from one spectral region to another, thereby surpassing the $4 n^{2}$ limit in a given region of interest [4].

In thin films, there is an additional constraint because the waves should interfere constructively after one round trip through the absorber. This gives rise to a number of discrete modes for both polarization directions; they are called guided modes because they propagate along the plane of the film. The solar cell is thus a somewhat unusual waveguide where the silicon film is the high index guiding medium and the back reflector is a cladding that doubles also as electric contact. Finally, the interface texture acts as coupler between the external radiation field and modes of the multilayer stack. If one of the constituent layers is metallic, the p-polarized mode of lowest order is no longer guided within one of the films, but along the metallic interface. This mode is then called surface plasmon polariton (SPP) and it was frequently suggested to be particularly interesting for light trapping [5, 6].

Stuart et al. took into account that a film can support only a limited number of waveguide modes at given wavelength and found that the absorption enhancement in thin films falls short of the $4 n^{2}$ bulk limit [7]. Schiff et al. extended this treatment recently to include the SPP mode, finding a significant enhancement beyond the bulk limit [8]. Yu et al. investigated the transition between discrete and dense modal structures of films with periodic interface texture [9, 10]. For wavelengths just above the onset of first order diffraction into air, their statistic treatment finds that the bulk limit of 
$4 n^{2}$ can be surpassed by factors of $\pi$ and $2 \pi / \sqrt{ } 3 \approx 3.6$ for interfaces with square and triangular periodicity, respectively. Periodicities in one dimension are somewhat academic, but useful for understanding; they have a "1D bulk limit" of $\pi n$ which can be surpassed by two, up to $2 \pi n$ for wavelengths directly above the grating period.

In this contribution, we discuss the waveguide modes of solar cell structures, taking into account the dispersion of the refractive indices in the individual layers as well as device-typical film thicknesses. The effect of the interface texture is discussed for periodic cases which can represent, for example, leading components of a spatial Fourier decomposition. The periodicity imposes Brillouin zones which directly yield conditions where the incident light can couple to guided modes. Finally, a statistical evaluation of the coupling conditions is used to obtain upper limits of absorption enhancement in realistic thin film solar cells.

\section{STATISTIC METHODS TO DETERMINE ABSORPTION ENHANCEMENT}

Derivations of the upper limit to absorption enhancement are based on the assumption that the interface texture distributes the incoming light uniformly among the available modes; details of the scattering mechanism are not taken into account. Yablonovitch et al. [3] gave a derivation based on the expression for the energy density in black body radiation which scales with $n^{2}$. By adding a perfect back reflector and full randomization by Lambertian scattering at the surface texture, they obtained an upper limit of $4 n^{2}$ for the absorption enhancement.

Yu et al. $[9,10]$ considered periodic interface texture and used discrete counting of modes, requiring different treatments for the regions within and without the absorbing film. In air, energy is carried away from the film by a number $N$ of diffraction modes; $N$ is at least one because of zero order reflection, additional modes appear for wavelengths shorter than the period. Within the film, the periodicity imposes forward and backward travelling Bloch waves. In out-of-plane direction, an analogous reciprocal coordinate can be constructed by fitting multiples of half the wavelength into the layer thickness; for consistency with later definitions, we define the order $m$ as number of nodes within the film, extending $m$ over the positive integers including zero, $m=0,1,2,3 \ldots$

For the following it is useful to define coordinates; 1D periodicity is assumed to be along the $x$ axis, 2D periodicity in the $x-y$ plane. The Bloch waves are constructed from discrete wave vectors $k_{\mathrm{x}}$ (1D) or $k_{\mathrm{x}}$ and $k_{\mathrm{y}}(2 \mathrm{D})$ which can assume negative and positive values whereas the out of plane coordinate is defined only for positive $k_{\mathrm{z}}$. Combination yields a reciprocal half plane for 1D periodicity and a reciprocal half space for 2D periodicity. The energy $E=\hbar \omega$ of the incident light defines a semicircle (1D) or hemisphere (2D) over the reciprocal coordinates. Then, the number $M$ of modes participating in a frequency range between $\omega$ and $\omega+\Delta \omega$ can be determined via the density of points in reciprocal $k$-space and the area (1D) or volume (2D) of a shell with thickness $\Delta \omega$. Finally, the absorption enhancement $F$ is defined by $[9,10]$ :

$$
F=\frac{2 \pi c}{n d \Delta \omega} \frac{M}{N}
$$

The derivation of eq. (1) relies on the assumption that the loss rate of guided modes can be related to the extinction coefficient of the absorber layer; an analysis of different solar cell configurations showed that this assumption is fulfilled for most of the relevant modes above $1.5 \mathrm{eV}$, but it is not valid in general [11]. Moreover, the theory applies to weakly absorbing materials which is the case for energies below $2 \mathrm{eV}$ in amorphous silicon. Showing wider energy ranges in this contribution serves illustration purposes, but conclusions should be drawn only for the relatively narrow light trapping region between 1.7 and $2 \mathrm{eV}$.

The enhancement factor for 1D periodicity is shown in Figure 1; periods of 560 and $833 \mathrm{~nm}$, a silicon thickness of $200 \mathrm{~nm}$ and $\Delta \omega$ equal to $0.1 \mathrm{eV}$ have been assumed. Because no depolarization is expected in 1D periodicity, s- and p-polarization are treated separately. Starting from low energies, the non-zero bars become gradually denser because with increasing energy more and more modes are allowed within the material. Sudden drops at wavelengths equivalent to the periods mark the onset of 
the $\pm 1^{\text {st }}$ order diffraction orders outside of the film, increasing $N$ from one to three. For illustration, the scale has been extended to include the onset of second order diffraction for the $833 \mathrm{~nm}$ period. For thicker films, the discrete data would smooth out and eventually approach the continuous sawtooth characteristic whose first and consecutive peak values are given by $2 \pi n, 4 \pi n / 3,6 \pi n / 5,8 \pi n / 7$, etc. The choice of a larger period smoothens the discrete data similarly by accommodating a larger number of Bloch waves, but additionally it squeezes the peaks of the sawtooth towards lower energies, shifting less and less noticeable steps into the shown energy range. Ultimately the 1D limit of $\pi n$ is reached.

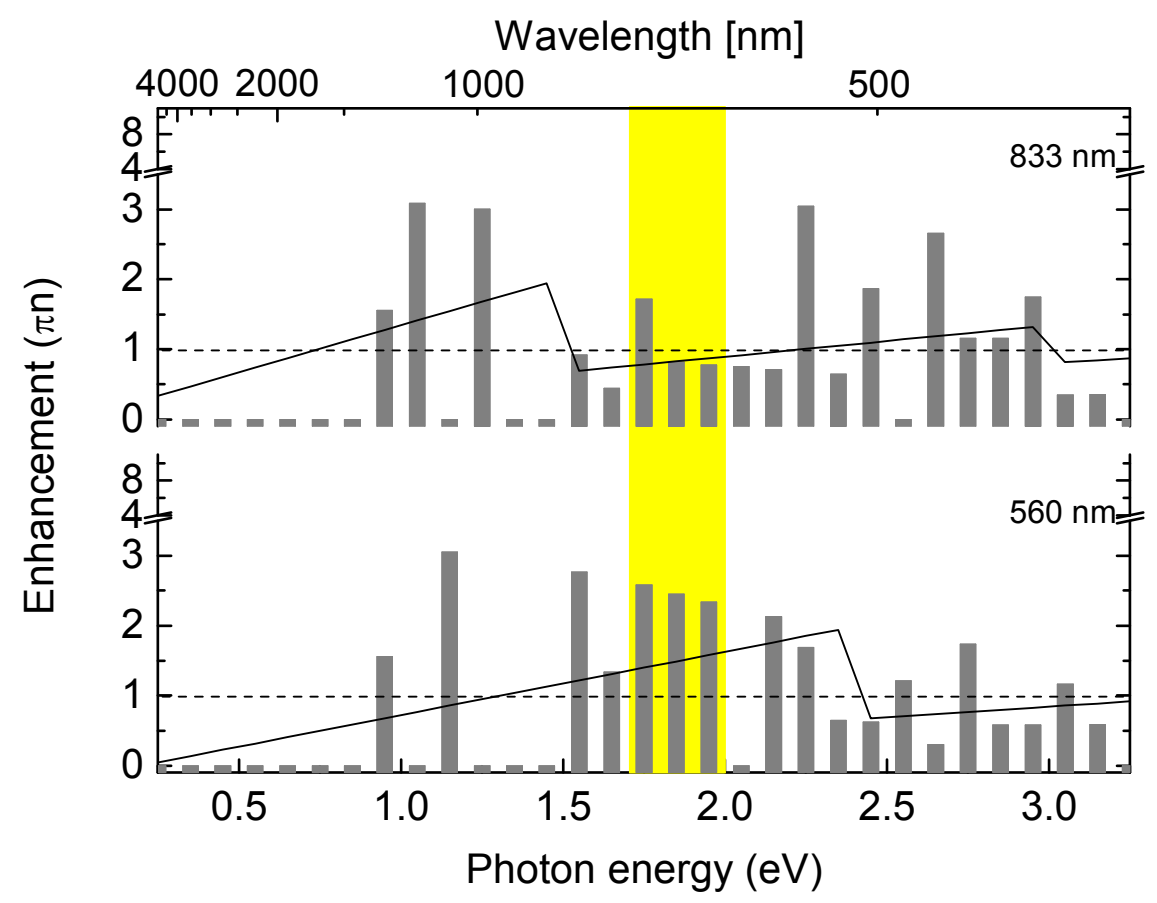

Figure 1: Absorption enhancement in $200 \mathrm{~nm}$ thick a-Si film between ideal mirrors and lateral periods of 560 (lower panel) and $833 \mathrm{~nm}$ (upper panel). The bars represent fully discrete counting of all individual resonances, the sawtooth line denotes continuous density within, but discrete diffraction orders outside of the film. The dashed line represents the 1D limit of $\pi n$. The shaded area denotes the light trapping region between 600 and $750 \mathrm{~nm}$.

Similar considerations apply to the case of interfaces with 2D periodicity, but the additional dimension yields a higher bulk limit of $4 n^{2}$ and the peak of $F$ for wavelengths just above the onset of first order diffraction is also higher. For the case of a square periodicity, it reaches a peak value of $4 \pi n^{2}$, for triangular periodicity the peak value is $8 \pi n^{2} / \sqrt{3}$ or approximately $4.6 \pi n^{2}[9,10]$. In the remainder of this contribution, we will restrict the discussion to the case of $1 \mathrm{D}$ interface textures along the $x$-direction, but the arguments apply equally to the general case of 2D textures in the $x y$-plane.

Alternative to counting in reciprocal space, the same results can be obtained from the dispersion diagram of $\omega(k)$ against $k$ [11]. After briefly discussing the properties of guided modes in terms of dispersion diagrams, we present examples where this description is advantageous. The requirement of nodes at the boundaries of the absorber layer restricts the allowed wave vectors in the out-of-plane direction $k_{z}$. This is illustrated in the inset of Figure 2 and can be used to define the dispersion relation of the waveguide modes which we will henceforth refer to as "idealized model":

$$
\omega(k)=c \cdot|k|=\sqrt{k_{x}^{2}+k_{z}^{2}}=\sqrt{k_{x}^{2}+((m+1) \cdot \pi / d)^{2}} \text {, with } m=0,1,2,3, \ldots
$$

The dispersion relations of the lowest three orders of eq. (2) are plotted in Figure 2. They show a cut-off at low energies, for high energies they approach asymptotically the dispersion relation of light in a medium which is given by $\omega(k)=c k / n$. As indicated by eq. (2), the characteristics extend towards negative $k_{\mathrm{x}}$ but negative orders of guided modes are again excluded.

Figure 2 illustrates the definition of Brillouin zones for a periodicity in $x$-direction. The counting of modes in the dispersion diagram starts by recognizing that energy and momentum must be conserved. The number of excited modes $M$ is then obtained by fixing an energy range of interest and 
counting all positions where any of the guided modes characteristics intersects with a predefined position within the Brillouin zones, e.g. the zone center.

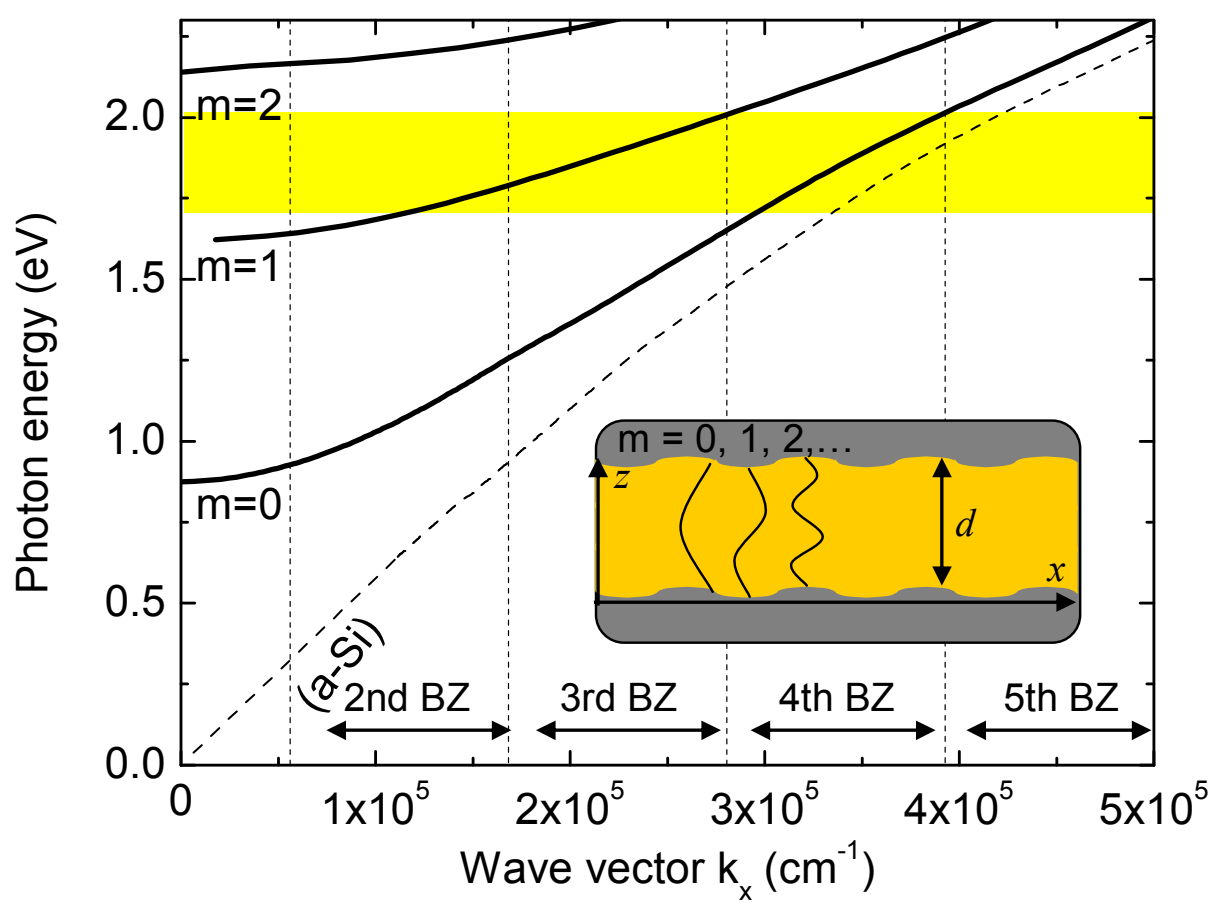

Figure 2: Dispersion relation of a metal/a-Si/metal waveguide eq. (2) (full lines). Vertical dashed lines illustrate Brillouin zones for a period of $560 \mathrm{~nm}$ in $x$-direction. The dashed line represents the dispersion relation of photons, $\omega(k)=c k / n$, i.e. propagation in the $x$-direction in bulk a-Si. The inset illustrates the structure and the field amplitude of the three lowest orders along the $z$-direction with nodes at the interfaces, the shaded are illustrates again the light trapping region.

For the shown example, the procedure does not hold any advantage over the counting scheme in reciprocal space, there is even a drawback because it cannot easily be made continuous as in reciprocal space by the definition of an energy surface. However, it does offer the possibility to introduce realistic dispersion relations. The model discussed above yields an illustrative definition of waveguide modes, but the underlying requirement of perfectly reflecting walls is not very realistic to begin with, and the only way to couple light into this structure is from the sides, by end-firing. This is clearly not envisioned for a large area application like solar cells.

\section{DISPERSION RELATIONS OF MULTILAYER STACKS}

Analytic solutions for dispersion relations exist only for simple situations whereas multilayer stacks must usually be treated numerically, particularly when the dispersion of the refractive indices is taken into account as well. Recognizing that eigenmodes of a stack of layers correspond to resonances and should manifest themselves as poles or singularities, Shakir et al. proposed to find eigenmodes by requesting zeros in the denominator of the Fresnel coefficient [12]. Fresnel coefficients of multilayer stacks are defined recursively, starting from the coefficients for the interface between two component media $i$ and $j$. For s- and p-polarization they are defined as follows:

$$
r_{i j}^{s}=\frac{S_{j}-S_{i}}{S_{j}+S_{i}} \text { and } r_{i j}^{p}=\frac{\varepsilon_{i} S_{j}-\varepsilon_{j} S_{i}}{\varepsilon_{i} S_{j}+\varepsilon_{j} S_{i}}
$$

The first and the last layers of the stack are assumed to be semi-infinite, thus applying to the case of a single interface between two materials. The propagation constants $S_{i}$ are defined for every 
individual layer $i$ in terms of its complex dielectric function $\varepsilon_{\mathrm{i}}$ and $k_{0}=2 \pi / \lambda$, the modulus of the wave vector in air:

$$
S_{i}^{2}=k_{0}^{2}-\varepsilon_{i} k_{x}^{2}
$$

For more than two layers, Fresnel coefficients are defined recursively via the single interface coefficients of eq. (3) and using an additional term for describing coherent propagation across the film thicknesses $d_{i}$ with the propagation constant $S_{i}$ :

$$
r_{1 \ldots n}=\frac{r_{12} \cdot r_{2 \ldots n} \cdot \exp \left\{-2 S_{2} d_{2}\right\}}{r_{12}+r_{2 \ldots n} \cdot \exp \left\{-2 S_{2} d_{2}\right\}}
$$

Eqns. (3) to (5) were used to determine the dispersion relation for a solar cell stack using five layers [11]; the silver layer is assumed semi-infinite and we considered a $60 \mathrm{~nm}$ thick $\mathrm{ZnO}$ buffer layer, a $200 \mathrm{~nm}$ thick amorphous silicon film, a $65 \mathrm{~nm}$ thick ITO front contact and an air space above. In the remainder, this will be called "realistic cell model". The resulting dispersion diagram is shown in Figure 3, the idealized modes are reproduced from Figure 2. Several differences are apparent; firstly, there is an area around the energy axis where no guided modes exist. This part of the diagram can be identified with a continuum of radiation modes and describes incident and reflected light. For any of these modes, only the in-plane component is plotted, corresponding to a dispersion relation of $\omega(k)=c k_{\mathrm{x}}=c k \sin \theta$. Thus, the characteristic of vertically incident light coincides with the energy axis and the shown light line of air represents grazing incidence under $90^{\circ}$. Secondly, all higher order guided modes cut off at the light line whereas the zero order modes extend to the origin at zero energy (not shown in the figure). Thirdly, the characteristics of a given mode order run at lower energy than the corresponding one of the idealized model. This is related to the open nature of the waveguide where a non-negligible part of the mode tails out of the high index guiding medium. The characteristics suggest thus an increased effective thickness. Finally, the two polarization directions are different for the same mode order and they show a cross-over (visible for $1^{\text {st }}$ and $2^{\text {nd }}$ order at 1.25 and $1.75 \mathrm{eV}$, respectively) [13].

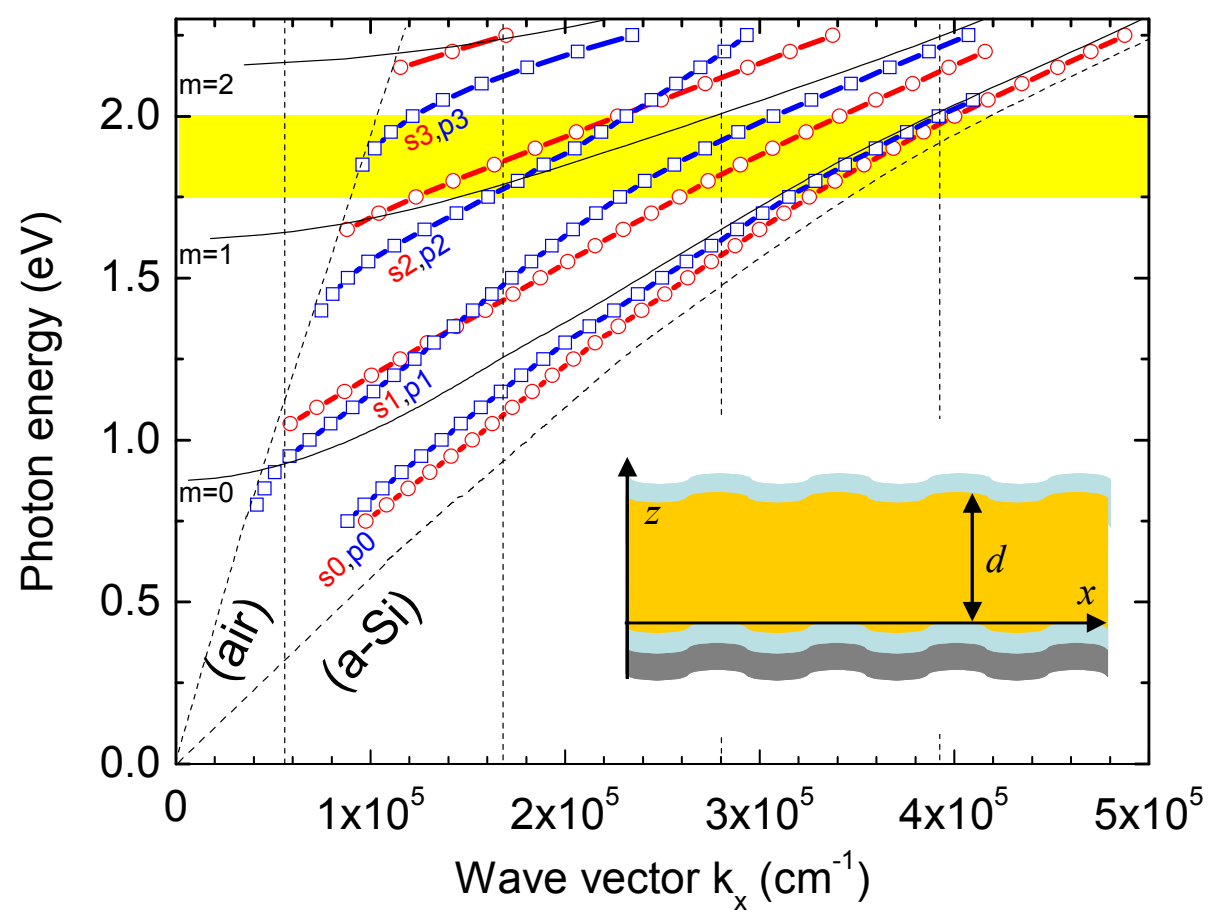

Figure 3: Dispersion diagram of a solar cell stack for s- (circles) and p-polarization (squares). The inclined dashed lines represent light lines of air and a-Si. Vertical lines denote the Brillouin zones for a period $P$ of $560 \mathrm{~nm}$. The full lines reproduce the idealized model from Figure 2. The inset illustrates the sequence of the layers (from top to bottom: air/ITO/a-Si/ZnO/Ag). 
The periodicity can couple the light line of air of a given angle of incidence from the radiation cone into the region beyond the $90^{\circ}$ horizon by a lateral shift of one or multiple reciprocal lattice vectors $G=2 \pi / P$, where $P$ is the period. For example, the light line of vertical incidence is thus reproduced in the centre of each Brillouin zone.

The dispersion relations shown in Figure 3 represent a solar cells stack that includes a thin layer of $\mathrm{ZnO}$ between the silver back reflector and the silicon absorber layer. Figure 4 shows how the omission of the $\mathrm{ZnO}$ buffer layer modifies the dispersion diagram. The most striking difference concerns the p0 mode which does no longer show the characteristics of guided modes which are restricted between the light lines of the lowest and the highest index material. Instead, the p0 mode crosses the silicon light line and flattens out just below $2 \mathrm{eV}$. It can thus be identified with the characteristic of a surface plasmon polariton (SPP), a particular form of guided mode where the electric field intensity is not concentrated within a guiding medium but at the interface between the metal and the adjacent dielectric material.

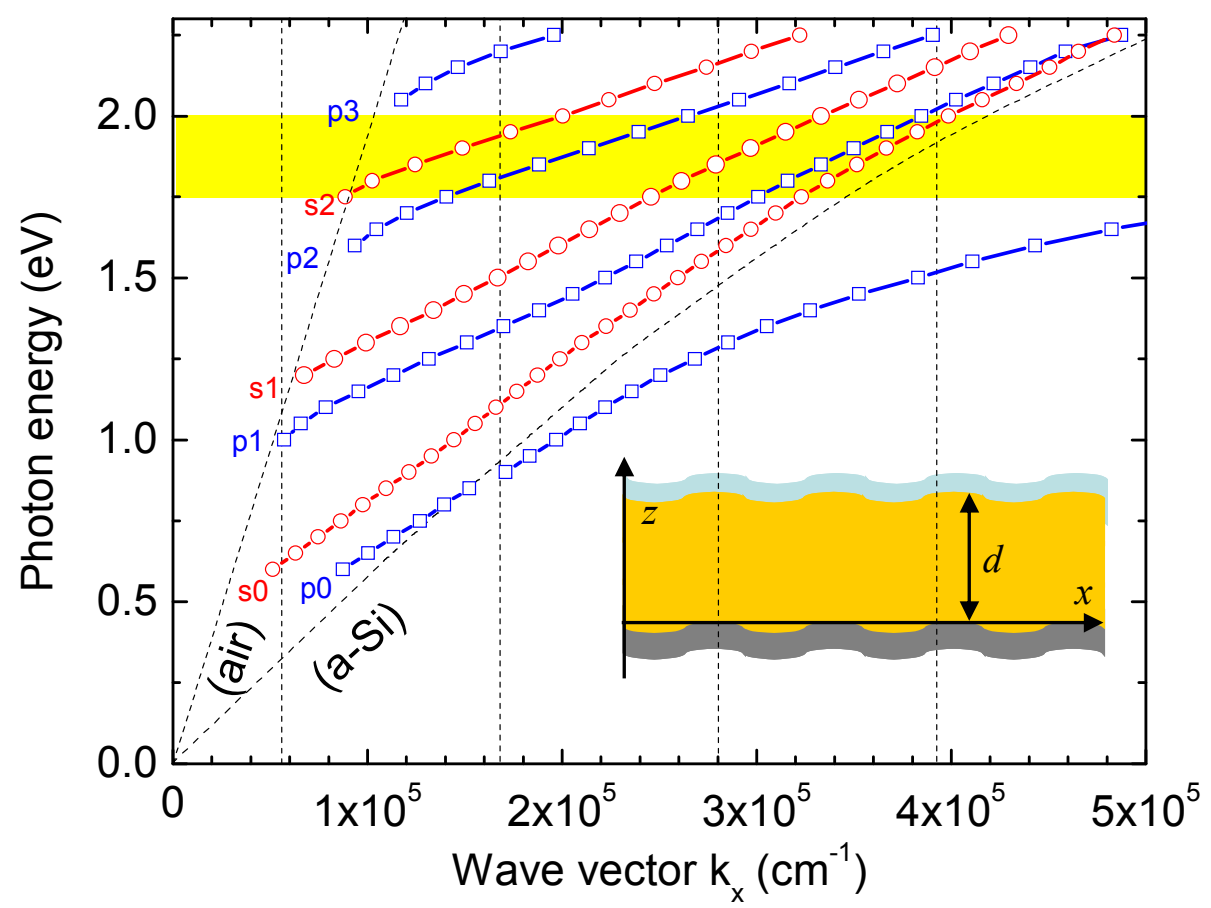

Figure 4: Dispersion diagram air/ITO/a-Si/Ag stack for s- (circles) and p-polarization (squares). The inclined dashed lines represent light lines of air and a-Si. Vertical lines denote the Brillouin zones for $560 \mathrm{~nm}$. The inset illustrates the sequence of the layers (from top to bottom: air/ITO/a-Si/Ag).

Initially intended merely as barrier against the diffusion of silver into silicon $[14,15]$, the $\mathrm{ZnO}$ buffer layer is still used in most of the modern solar cell architectures with metallic contacts [16-18], although it has been proposed to replace it by alternate low index materials like doped silicon oxide $[19,20]$. A comparison of Figures 3 and 4 shows that the $\mathrm{p} 0$ mode is no longer a surface plasmon polariton as soon as the buffer layer is included; rather, it transforms into a regular waveguide mode whose dispersion relation is above the silicon light line [13].

\section{ABSORPTION ENHANCEMENT IN SOLAR CELLS}

Our alternate counting procedure was applied to the realistic dispersion diagrams shown in Figures 3 and 4. We assumed perpendicular incidence and periodicities of $560 \mathrm{~nm}$ and $833 \mathrm{~nm}$. The statistical evaluation of the full solar cell stack is shown in Figure 5. As already seen for the idealized model, the period of $833 \mathrm{~nm}$ is not very well suited for amorphous silicon absorbers because its 
enhancement factor dips exactly in the light trapping region of this material. In contrast, the period of $560 \mathrm{~nm}$ promises much better enhancement in this spectral range. Owing to the increased effective thickness of the open waveguide structure, all resonances in the low order Brillouin zones occur at lower energy. Thus, the solar cell stack yields considerably higher enhancement than the results of the idealized case in Figure 1.This finding will be discussed in more detail in the next section.

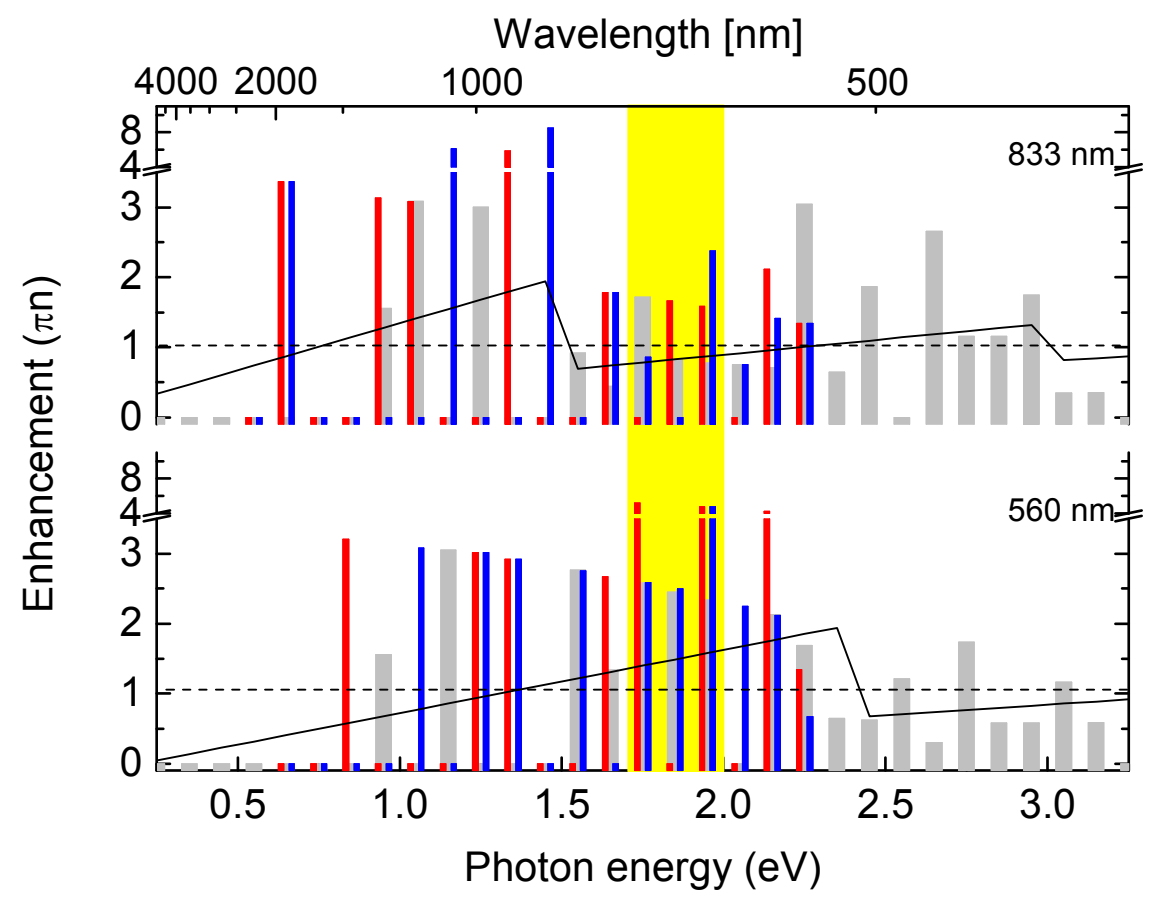

Figure 5: Enhancement factors using the dispersion relation of the solar cell shown in Figure 3. The left and right narrow bars represent s- and p-polarization, respectively. The wide bar in the background and the lines reproduce results of Figure 1.

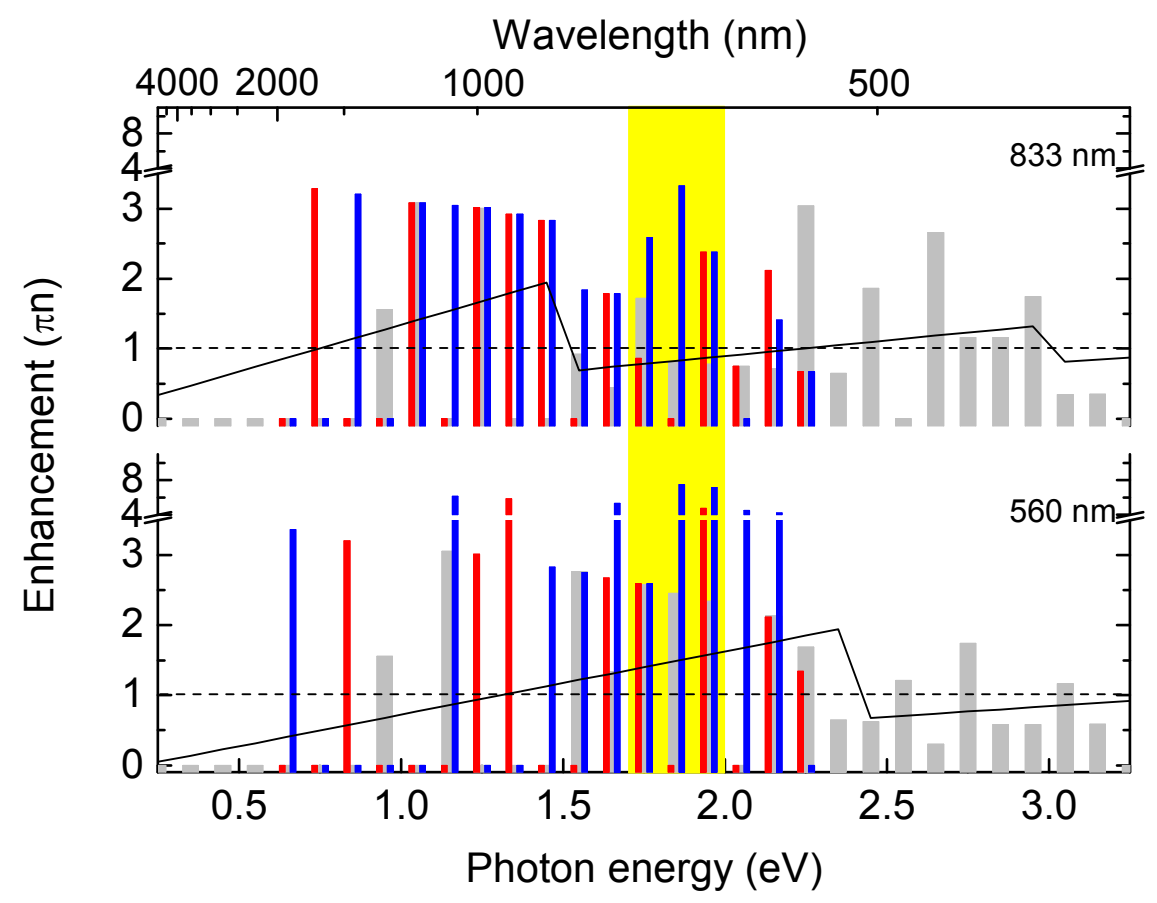

Figure 6: Enhancement factors using the dispersion relation of the plasmonic structure shown in Figure 4. Bars and lines have the same meaning as in Figure 5. 
From the definition of our counting procedure, the flattening of the $\mathrm{p} 0$ characteristic promises a significantly increased number of resonances for energies between 1.5 and $2 \mathrm{eV}$. Indeed, Figure 6 shows that the enhancement in this region is much higher for p-polarization than for s-polarization. The enhancement is more than doubled in this region, compensating completely for the deficit of the $833 \mathrm{~nm}$ periodicity. For the $560 \mathrm{~nm}$ periodicity the enhancement around $2 \mathrm{eV}$ averages to six-fold increase over the $\pi \mathrm{n}$ bulk limit because there is the additional benefit of low $N$ as only one single diffraction mode is allowed.

\section{FIELD DISTRIBUTION}

The findings of the previous section are promising, but the extension from the completely closed waveguide of the idealized model to the open structure necessitates a more detailed analysis. Because the field amplitude can extend out of the waveguide, only the actually confined part of the electric field should be taken into account; Stuart et al. suggest a weighting on the basis of phase and group velocities in a guiding medium on a substrate [7], Yu et al. propose the ratio between modal attenuation and the absorption coefficient of silicon [10]. Other definitions of confinement factors exist in the waveguide literature, e.g. [21, 22].

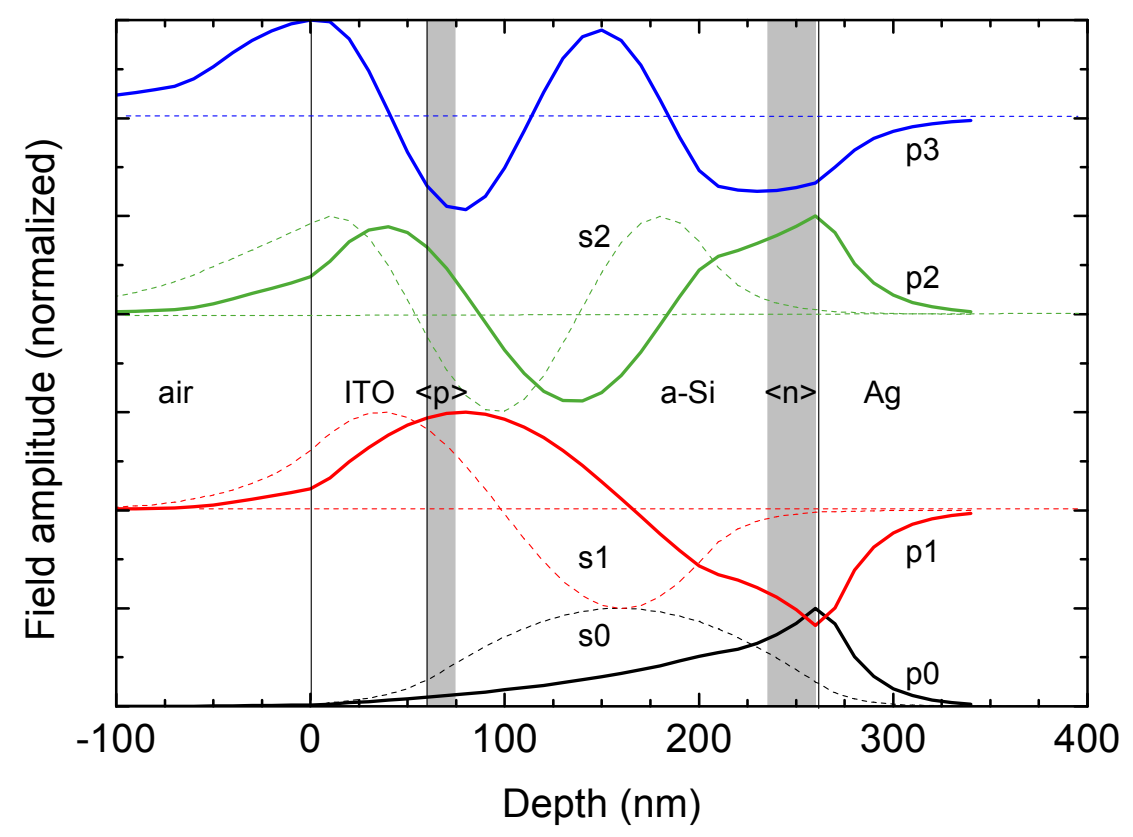

Figure 7: Electric field amplitudes of the lowest order guided modes in the structure without $\mathrm{ZnO}$ buffer at an energy of $2 \mathrm{eV}$ (note that there is no s3 mode at this energy). Dashed and full lines illustrate s- and p-modes, respectively. The shaded areas denote the approximate thicknesses of 10 to $15 \mathrm{~nm}$ and 20 to $25 \mathrm{~nm}$ of p- and ndoped layers, respectively.

For illustration of this point, Figure 7 plots the field profiles of the solar cells stack without the $\mathrm{ZnO}$ buffer layer for s- and p-modes at $2 \mathrm{eV}$. For the zero order modes there is a clear difference between the polarization directions; as mentioned already, the SPP mode assumes its maximum field amplitude at the interface between silicon and silver while the s0 mode peaks almost exactly in the middle of the absorber layer. For the first order modes the situation slightly different; the p1 shows again a high amplitude at the interface to the back contact, but the maximum towards the front of the device is still within silicon. In the s1 mode, this peak is exactly in the ITO layer, any absorption associated with this part will not contribute to the photocurrent.

Even if the fields assume their extreme values within the silicon film, there is a second concern; the presence of the doped layers was neglected for the calculation of the dispersion relations 
on the basis that their refractive indices differ very little from intrinsic silicon. However, absorption in these layers does not contribute to the photocurrent. The shaded areas in Figure 7 illustrate the position and typical thickness of $n$ - and p-doped layers, showing that the p-polarized modes are likely to suffer from losses because all of them show significant field amplitude within the n-doped layer. We plan to include a weighting of the different resonances into the counting procedure in our future work.

\section{CONCLUSIONS}

We investigated the effect of a periodic interface texture on the upper limits of absorption enhancement in amorphous silicon solar cells. We proposed a statistic procedure that can take into account the actual dispersion relation of the eigen modes, providing a tool to asses realistic structures like full solar cell configurations and back reflector strategies with plamsonic effects. For the interface between silicon and silver, the resonance character of the latter promises extraordinary enhancement in the desired spectral region of weak absorption. However, an analysis of the field amplitude profile across the multilayer stack suggests that the potential of the surface plasmon polarition (SPP) mode is actually rather limited.

\section{ACKNOWLEDGEMENTS}

This work was funded by the European Union within the project Si-Light (Contract No 241277), by the Swiss National Science Foundation (Contract Nos. 2000021_125177/1 and 200020_137700/1) and by the Swiss Federal Office for Energy (Contract No. 101191).

\section{REFERENCES}

1. D. E. Carlson and C. R. Wronski, "Amorphous Si Solar Cell" Applied Physics Letters 28(11), p. 671-673 (1976)

2. H. W. Deckman, C. R. Wronski, H. Witzke, and E. Yablonovitch, "Optically enhanced amorphous silicon solar cells" Applied Physics Letters 42(11), p. 968-970 (1983)

3. E. Yablonovitch and G. D. Cody, "Intensity enhancement in textured optical sheets for solar cells" Electron Devices, IEEE Transactions on 29(2), p. 300-305 (1982)

4. P. Sheng, A. N. Bloch, and R. S. Stepleman, "Wavelength-selective absorption enhancement in thin-film solar cells" Applied Physics Letters 43, p. 579 (1983)

5. H. A. Atwater and A. Polman, "Plasmonics for improved photovoltaic devices" Nature materials 9(3), p. 205-213 (2010)

6. V. E. Ferry, J. N. Munday, and H. A. Atwater, "Design considerations for plasmonic photovoltaics" Advanced Materials 22, p. 4794 (2010)

7. H. R. Stuart and D. G. Hall, "Thermodynamic limit to light trapping in thin planar structures" JOSA A 14(11), p. 3001-3008 (1997)

8. E. Schiff, "Thermodynamic limit to photonic-plasmonic light-trapping in thin films on metals" Journal of Applied Physics (to be published)

9. Z. Yu, A. Raman, and S. Fan, "Fundamental limit of light trapping in grating structures" Optics Express 18(103), p. A366-A380 (2010)

10. Z. Yu, A. Raman, and S. Fan, "Fundamental limit of nanophotonic light trapping in solar cells" Proceedings of the National Academy of Science 107(41), p. 17491 (2010)

11. F.-J. Haug, K. Söderström, A. Naqavi, and C. Ballif, "Resonances and absorption enhancement in thin film silicon solar cells" Journal of Applied Physics 107, p. 044504 (2011)

12. S. Shakir and A. Turner, "Method of poles for multilayer thin-film waveguides" Applied Physics A: Materials Science \& Processing 29(3), p. 151-155 (1982) 
13. F.-J. Haug, T. Söderström, O. Cubero, V. Terrazoni-Daudrix, and C. Ballif, "Influence of the $\mathrm{ZnO}$ buffer on the guided mode structure in $\mathrm{Si} / \mathrm{ZnO} / \mathrm{Ag}$ multilayers " Journal of Applied Physics 106, p. 044502 (2009)

14. C. Kothandaraman, T. Tonon, C. Huang, and A. E. Delahoy. Improvement of a-Si:H p-i-n devices using zinc oxide based back reflectors. in Proc. MRS Spring Meeting. 1991. San Francisco: MRS. p. 475-480

15. R. Ross, R. Mohr, J. Fournier, and J. Yang. Status of fluorinated amorphous silicon-germanium alloys and multijunction devices. in Proc. 19th IEEE PVSC. 1987. New Orleans. p. 327-330

16. R. Biswas, D. Zhou, B. Curtin, N. Chakravarty, and V. Dalal. Surface plasmon enhancement of optical absorption of thin film a-Si:H solar cells. in Proc. 34 th IEEE PVSC. 2009.

Philadelphia. p. 557

17. V. E. Ferry, M. A. Verschuuren, H. Li, R. E. I. Schropp, H. A. Atwater, and A. Polman, "Improved red-response in thin film a-Si: H solar cells with soft-imprinted plasmonic back reflectors" Applied Physics Letters 95, p. 183503 (2009)

18. M. Naughton, K. Kempa, Z. Ren, Y. Gao, J. Rybczynski, N. Argenti, W. Gao, Y. Wang, Y. Peng, and J. Naughton, "Efficient nanocoax-based solar cells" Physica Status Solidi - Rapid Research Letters 4, p. 181-183 (2010)

19. F.-J. Haug, T. Söderström, O. Cubero, V. Terrazzoni-Daudrix, and C. Ballif, "Plasmonic absorption in textured silver back reflectors of thin film solar cells" Journal of Applied Physics 104, p. 064509 (2008)

20. P. Delli Veneri, L. V. Mercaldo, and I. Usatii, "Silicon oxide based n-doped layer for improved performance of thin film silicon solar cells" Applied Physics Letters 97, p. 023512 (2010)

21. W. Anderson, "Mode confinement and gain in junction lasers" Quantum Electronics, IEEE Journal of 1(6), p. 228-236 (1965)

22. Y. Z. Huang, Z. Pan, and R. H. Wu, "Analysis of the optical confinement factor in semiconductor lasers" Journal of Applied Physics 79, p. 3827 (1996) 